\title{
Rules Extraction for Fuzzy Control Systems
}

\author{
Yung-Yaw Chen
}

Department of Electrical Engineering

National Taiwan University

Taipei, Taiwan, R.O.C.

\section{ABSTRACT}

A method of extracting the control rules for a fuzzy controller is proposed in this paper. The method successfully constructs the rule base for a fuzzy controller, which is usually provided by human experts. By using the concepts of cell state space and perform ance evaluation, an optimization algorithm is able to achieve the proper control of a process. The control rules can then be retrieved from the output of this algorithm. This method extracts qualitative control rules from the quantitative processes. The qualitative description of fuzzy control rules is important for its robustness and flexibility.

\section{Introduction}

One of the characteristics of fuzzy control is the total dependency on experts' knowledge for its controller design. Human dency on experts knowledge for its controller design. Humat accomplish difficult tasks. The powerful summarizing ability of accomplish difficult tasks. The powerful summarizing ability of
buman experts contributes greatly to the excellent performance of a fuzzy controller. Moreover, a fuzzy controller is designed to grasp the key factors for controlling a certain process with only a few number of rules and without too much mathem atical details. Therefore, its perform ance is usu ally not very accurate and is not expected to be so either. The inaccuracy of its performance is compensated by the simplicity of the controller design.

However, the strength of this approach, the usage of experts' knowledge, also limits the applicable domains of fuzzy control. For some cases, there may not be any skilled operator or expert available to provide necessary information for the controller design. For example, a very complex heat dissipation problem in NASA's space station requires extremely complicated modeling and computation if handled by conventional methods. A fuzzy controller, on the other hand, may be a reasonable solution. However, the design process has to be done without a truly skilled expert. The automation for fuzzy controller design therefore becomes a very important issue. It would be valuable if we could develop a method which can systematically extract similar control rules from a process as the human experts'.

A linguistic self-learning process controller was developed by Procyk and Mamdani[1], which utilized a performance measurement decision table given by the experts to achieve self-learning behavior. However, they did not explicitly formulate the rules to form a rule-base. Tanscheit and Scharf 21 and Shao [3] applied the on a che crosults. for fuzzy control based on fuzzy relational equations.

The approach proposed in this paper is based on the usage of the cell state space and the performance evaluation. The concept of the cell state space has been very useful in the an alysis of fuzzy dynamical system s[5-7]. The basic idea of the cell state space is to represent a state space, which contains infinite number of points by finite number of cells. The dynamics of the real process is then approximated by a cell-to-cell mapping $[8,9]$. We first seek the proper control action on each cell on the partitioned cell state space by evaluating a performance index associated with each cell and each control action. Then we transform the result into a rulebased form and hence accomplish the rules extraction.

\section{Description of the method}

To simplify the notation, we shall consider only two dimensional state space in this paper. Higher dimensional cases can be easily extended.

\subsection{Cell Control System}

The block diagram of a cell control system which consists of a plant and a cell controller is shown in Figure 1.

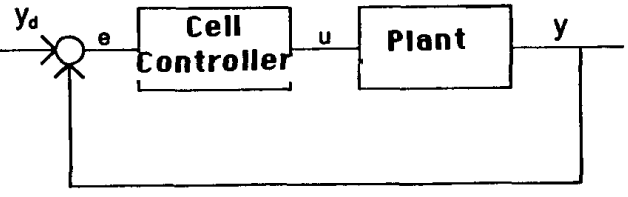

Figure 1 Cell Control System

where $e$ is the error, $u$ is the input of the plant, $y$ is the output of the plant and $y_{d}$ is the desired output.

Cell State Space

Assume that the state space $X=X_{1} \times X_{2}$ has a domain of interest $D=D_{1} \times D_{2}=\left[d_{11}, d_{12}\right] \times\left[d_{21}, d_{22}\right]$. The domain of interest $D$ is partitioned into $n_{1} \times n_{2}$ number of cells, called regular cells. The region outside the dom ain of interest is called the sink cell. The state space is, therefore, partitioned into $n \times n$ regular cells and one sink cell, which together constitute the cell state space. Any state $x \in X$ must belong to a cell $z$ in
the cell state space.

Cell Controller

The cell controller is constructed by associating a real input with each cell in the cell state space. Whenever, the state trajectory enters a cell, the associated input is fired.

\section{Performance Evaluation}

A performance index $f\left(x_{1}, x_{2}\right)$ which is a function of the state variables is chosen to be the cost function for finding the optimal input for each cell. It can be any Lyapunov-like function, such as the $l-2$ norm of the state variables. The basic idea is to find a real-valued input which will minimize the performance index function, i.e. drive the system states toward the desired goal.

Control Input Modification

In order to find the proper input associated with each cell, several parameters are assigned to each cell and are described in the following.

(1) Value of the input $u(z)$

CH2809-2/89/0000-0526\$1.00 1989 IEEE 
(2) Sign of the change of the input value for each iteration, $\operatorname{sign}(z)$

(3) Size of the change of the input value for each iteration, $\Delta u(z)$ (4) Change of the performance index for each cell,
$\Delta f(z)=f_{\text {new }}-f_{\text {old }}$

All the parameters will be assigned some constant values initially, such as $\operatorname{sign}(z)=1, \Delta u(z)=1.0, \Delta f(z)=0.0$, and $\Delta^{2} f(z)=0.0$, for all $z$. Then the magnitude of the input for each cell will be modified based on the following algorithm:

(1) if $\Delta f<0.0$, then $u_{k+1}=u_{k}+s i g n_{k} \times \Delta u_{k}$

(2) if $\Delta f=0.0$, then $u_{k+1}=u_{k}$

(3) if $\Delta f>0.0$, then

$s i g n_{k+1}=-\operatorname{sign}_{k}, \quad \Delta u_{k+1}=\frac{1}{k+1}$ × $\Delta u_{0}$

and

The algorithm described above is basically a simple optimization algorithm using maximum descent method. The purpose of the algorithm is to derive the control input on each cell to construct a stablizing cell controller. It is possible for this algorithm to be trapped in some local minimum points. Some clever heuristics can be used to avoid the problem in most cases.

\subsection{Rules Extraction}

The purpose of rules extraction is to derive proper control rules directly from the inputs associated with each cell instead of from the knowledge of experts. The basic idea is to divide the whole state space into a number of overlapping regions such that each region is represented by one fuzzy control rule. Each rule is of the form:

If $x_{1}$ is small, and $x_{2}$ is medium, then $u$ is small.

where small and medium are fuzzy linguistic terms.

Idealy, we should consider the partitioning of the state space based on the distribution of the magnitude of the cell inputs to derive a rule base. However, the problem will be simplified if we start with a pre-specified precondition part and focus on the derivation of the action part of the rules. The first approach involves more techniques in data transform ation and is still under investigation by the author. In this paper, we will only discuss the tion by the author, In this paper,
approach with known preconditions.

Firstly, assume that we have three linguistic terms, namely small, medium, and large, for the two state variables $x_{1}$ and $x_{2}$ respectively. Then there will be $3 \times 3$ control rules in the rule base. The output of the fuzzy controller, with the commonly used weighted average method $[10]$, is given as:

$$
u=\frac{w(1) u(1)+\cdots+w(9) u(9)}{w(1)+\cdots+w(9)}
$$

where $w(i), i=1, \ldots, 9$ are the matching membership values for the rules, and $u(i), i=1, \ldots, 9$ are their output values.

The problem of rules extraction, or the determination of the action part, is actually to find the proper values of $u(i), i=1, \ldots, 9$ such that Eq. (1) holds when given a state $\left(x_{1}, x_{2}\right)$.

\section{Credit Assignment Problem}

A common difficulty encountered by many learning algorithms is the credit assignment problem In our case, we need to know what are the contributions of each rule to the output of the controller. It is most likely that there is no unique solution for this controller. It is most likely that there is no unique solution for this
problem. The method we proposed based on the assumption of the completeness of the fuzzy controller, i.e. there is always a dominant rule at each instant. It is also reasonable to assume that the output of the controller is decided mostly by the dominant rule and the contribution of each rule is proportional to the membership value associated with each rule. i.e.

$$
u(i)=w(i) t, \quad i=1, \ldots, 9
$$

Since the values of $w(i), i=1, \ldots, 9$ and $u$ are known once the state $\left(x_{1}, x_{2}\right)$ is given, we can derive $t$ by:

$$
t=\frac{u(w(1)+\cdots+w(9))}{w(1)^{2}+\cdots+w(9)^{2}}
$$

Therefore,

$$
\begin{aligned}
& u(1)=w(1) t=\frac{u w(1)(w(1)+\cdots+w(9))}{w(1)^{2}+\cdots+w(9)^{2}} \\
& u(9)=w(9) t=\frac{u w(9)(w(1)+\cdots+w(9))}{w(1)^{2}+\cdots+w(9)^{2}}
\end{aligned}
$$

Finally, the control rules extraction from the cell controller is achieved by the following procedures:

(1) Represent each cell $z$ by its center point $x_{c}$.

(2) Derive the membership value associated with each rule with the state $x_{c}$ for each cell.

(3) Use Eq. (1)-(5) to derive the ( $w(i), u(i)), i=1, \ldots, 9$ for each cell.

(4) Connect all the $(w, u)$ points to form the membership curve of the control input for each rule and hence form the control rule-base.

\section{Simulation and Discussion}

Preliminary simulation result shows that the proposed method is able to construct a rule base which is similar to an existing working fuzzy controller designed by experts. However, the method will work best if the pre-condition part of the rules are properly chosen. Improper partition of the state space may create inconsistent result on the membership function of the action variable. It is obvious that further study is required to include the pre-condition part into consideration.

\section{Conclusions}

The proposed method aims to extract fuzzy control rules for a process without the help from any expert. It is basically an offline process. However, because of the increasing processing speed, the method can be extended to become a real-time process, i.e. to become a real-time learning controller. One possible direction will be to combine the neural network technique with the fuzzy rulebase structure to accomplish the learning task. Further studies are conducted by the author and will not be discussed in this paper.

\section{References}

[1] T. J. Procyk and E. H. Mamdani, "A Self-Organizing Linguistic Process Controller", Autom atica 15, pp. 15-30, 1979.

[2] R. Tanscheit and E. M. Scharf, "Experiments with the use of $a$ $R$ ule-based Self-organizing Controller for Robotics Applications", Fuzzy Sets and Systems 26, pp. 195-214, 1988.

[3] S. Shao, "Fuzzy Self-organizing Controller and its Application for Dynamic Processes", Fuzzy Sets and Systems 26, pp. 151-164, 1988.

[4] E. Czogala and W. Pedrycz, "Fuzzy Rules Generation for Fuzzy Control", Cybernetics and Systems: An International Journal, Vol. 13, pp. 275-293, 1982.

[5] Y.-Y. Chen, "The Analysis of Fuzzy Dynamic Systems Using Cell-to-Cell Mapping", Proceedings of 1988 IEEE Systems, $M a n$, and Cybernetics Annual Conference.

[6] Y.-Y. Chen and T.-C. Tsao, "A New Approach for The Global Analysis of Fuzzy Dynamic Systems", Proceedings of the 27 th Conference on Decisions and Control, 1988.

[7] Y.-Y. Chen and T.-C. Tsao, "A Description of the Dynamical Behavior of Fuzzy Systems", IEEE Transaction of Systems, $\mathrm{Man}$, and Cybernetics, July, 1989.

[8] C. S. Hsu, "A Theory of Cell-to-Cell Mapping Dynamical Systems", ASME Journal of Applied Mechanics, Vol. 47, pp. 931-939, 1980.

[9] C. S. Hsu and R. S. Guttalu, "An Unravelling Algorithm for Global Analysis of Dynamical Systems: An Application of Cellto-Cell Mappings', ASME Journal of Applied Mechanics, Vol. 47, pp. 940-948, 1980.

[10] Sugeno, M., "An Introductory Survey of Fuzzy Control", Information Sciences, Vol. 36, pp.59-83, 1985. 